\title{
A MapReduce-based Adjoint method for preventing brain disease
}

\author{
Manal Zettam*, Jalal Laassiri and Nourddine Enneya
}

${ }^{*}$ Correspondence:

zettammanal@gmail.com

Informatics, Systems

and Optimization Laboratory,

Department of Computer

Science, Faculty of Science,

Ibn Tofail University, Kenitra,

Morocco

\begin{abstract}
In this paper, we present a statistical model performed on the basis of a patient dataset. This model predicts efficiently the brain disease risk. Multiple regression was used to build the statistical model. The least squares estimation problem usually used to estimate the parameters of regression model is solved via parallelized algebraic Adjoint method. As the parallelized algebraic Adjoint method is not the only Mapreduce-based method used to solve the least square problem, experimentations were carried out to classify the Adjoint method amongst the other methods. The calculated job completion time shows the competitive trait of the Mapreduce-based Adjoint method.
\end{abstract}

Keywords: Brain disease, Adjoint method, Multiple regression, MapReduce

\section{Introduction}

According to Li et al. [1], Kumar and Hancke [2], Luke and Stamatakis [3], quantifying disease risks of individuals is a relevant aspect of e-Health. In literature, studies concerning the brain disease risks commonly focus on:

- Neuro-imaging $[4,5]$,

- Blood-based biomarkers [6],

- And predictive characteristics of the Genetics-based Biomarker Risk Algorithm [7].

Further studies highlight the relevance of physical exercises and diet to prevent Alzheimer's disease [8-11]. Moreover, the relationship between burden and Alzheimer's disease is pinpointed in $\mathrm{Bu}$ et al. [12], the one binding bacterial infection and Alzheimer's disease is identified in Maheshwari and Eslick [13] and finally the one relating the Lyme and Alzheimer's diseases is reported in MacDonald [14].

To the best of our knowledge, existing studies of Alzheimer's disease prediction do not rely on a software solution based on factors such as ages, daily work's hours, and the existence of a parent with Alzheimer's disease. Therefore, we propose a solution which receives a dataset of patients with a variable number of attributes and then constructs a statistical model to spot eventual Alzheimer's disease patients. We also parallelize the Adjoint method via MapReduce. The parallelized algebraic Adjoint method has been presented briefly for the first time by our previous work in Zettam et al. [15].

(c) The Author(s) 2018. This article is distributed under the terms of the Creative Commons Attribution 4.0 International License (http://creativecommons.org/licenses/by/4.0/), which permits unrestricted use, distribution, and reproduction in any medium, provided you give appropriate credit to the original author(s) and the source, provide a link to the Creative Commons license, and indicate if changes were made. 
The proposed solution estimates the Alzheimer's disease risk based on a statistical model. Statistical models for prediction can be discerned in three main classes: regression, classification, and neural networks [16].

Regression analysis is one of the most predominant empirical tools. It is used to predict the unknown value of a variable from the known value of one or more variables also called the predictors [17]. The simple, multiple and logistics regression are the most used forms of regression in the literature [18]. The adequate choose of the regression model form depends on the number of predictors and the type of the outcome variable. The book referenced in Hosmer and Lemeshow [19] presents a detailed overview of logistic regression and its applications. In their part, the references [20,21] give detailed overviews of simple and multiple regressions with examples of their applications in real life problems. In medical field, several studies used the regression model such as predicting long-term mortality in oesophageal [22] and relative survival in cancer registries [23].

Classification has two distinct meanings. The first type is known as unsupervised learning (or clustering), the second as supervised learning [17]. In the statistical literature, supervised learning is usually referred to as discrimination, by which is meant the establishing of the classification rule from given correctly classified data [17]. Chatap and Shrivastava [24] presented a detailed survey on classification methods involved in medical field such as the CART method [25], The CSO decision tree algorithm [26], Chi squared automated interaction detection [27], Quick, Unbiased, Efficient, Statistical Tree (QUEST) [28], Discriminate Analysis [29]. Further information can be found in Michie et al. [17].

The term neural network encompasses a large class of models and learning methods. Neural network method is a nonlinear statistical model. Neural network was developed decades ago by scientists attempting to model the learning process of human brain [30]. The most known method of neural network is called the single hidden layer backpropagation network. The discovery of back propagation in the late 80 s by Rumelhart et al. [31] was an impetus to the adoption of neural network in several fields such as medical field. In this field, the neural network methods have proven their efficiency as a diagnosing tool. Indeed, since the study performed by Szolovits et al. [32] many studies have been published such as colorectal cancer [33], multiple sclerosis lesions [34], colon cancer [35], pancreatic disease [36], gynecological diseases [37], and early diabetes [38]. Readers may refer to Amato et al. [39] for more details.

Other statistical models which not fit in the three main classes are used in the prediction literature such as those presented in Cesa-Bianchi and Lugosi [40] and Chen et al. [41]. Those models differ from the ones we presented above.

As stated before, the choice of a suitable statistical model depends on the type of predictors and the nature of the outcome. Furthermore, the use of variance analysis instead of regression to provide a quantitative outcome is a common issue pointed out by a number of statisticians such as Anderson et al. [42], Tribout [43]. These authors clearly report the main differences between regression and variance analysis. In addition, the reference [43] claims that some of software solutions aiming at facilitating their use combine regression and variance analysis under the acronym ANOVA.

In this study, the regression model is used to perform the prediction model due to the nature of predictors and outcome variable. The rest of the paper is organized as follows. The second section addresses the case study. This section is discerned in many 


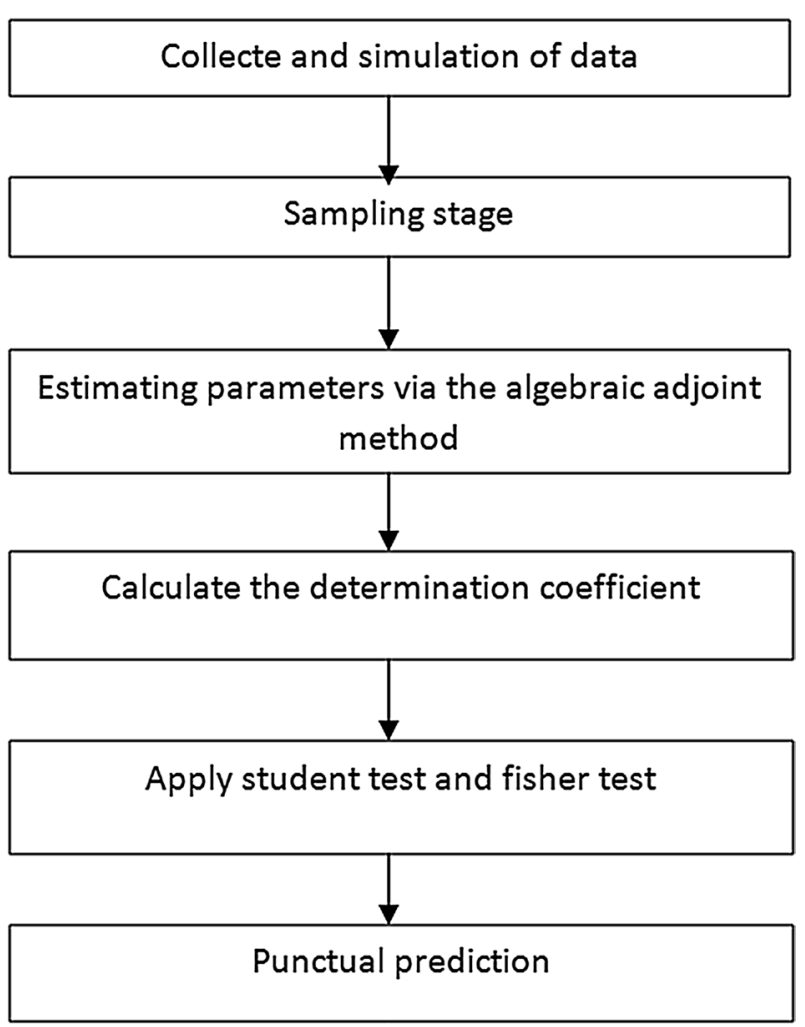

Fig. 1 Chart flow of the proposed software solution

sub-sections that present the variables used for modeling, detail the sampling stage, relate the application of multiple regression, give a brief overview of the Adjoint method used to solve the least squares estimation problem and introduce the MR-AM method. Then, the third section presents the technique used to evaluate the strength of the resulting model. Finally, the last section sums up the current work.

\section{The Alzheimer's disease prediction case study}

In this paper, a case study is presented on predicting patients with Alzheimer's disease risk. Unfortunately, none of the previously presented studies in literature provides sufficient data to perform our study. Therefore and instead of collecting data from literature, a simulated dataset is generated. Other studies in literature were based on simulated datasets such as Tresch et al. [44], Giglio et al. [45], Murray et al. [46]. To define the predictors of the current study, we were based on previous studies highlighting the importance of physical exercises, feeding, quality of life and existence of a parent with Alzheimer disease. Based on those factors, the aim of the study is to give a percentage of Alzheimer's disease risk for each individual in a population. Since, multiple predictors are involved and the outcome we aim to obtain is quantitative, multiple regression is the most suited statistical model to perform the study [16]. The theoretical bases of regression are explained thereafter. The steps undertaken in this study are presented in Fig. 1.

The regression analysis is a statistical model that indicates how the variables are related on the basis of an equation. Formally, the variable we are trying to predict is 
called dependent variable, the variable or variables to predict the value of the dependent variable are called independent variables (predictors). The simple regression is a regression with single independent variable. The multiple regression is a regression with multiple independent variables. The procedures to accomplish simple and multiple regression are in somehow similar.

\section{The simple regression}

Assuming the case where the Alzheimer's disease risk is predicted on the base of one predictor, for instance, the age of a patient. The population undertaken in this study is a population of patients recorded in a dataset. The aim is to predict the percentage of Alzheimer's disease risk denoted $y$ on the base of the patient age denoted $x_{1}$.

The Eq. (1) describes the relation binding $x$ and $y$ with an error term denoted $\varepsilon$, corresponds to a regression model. The model used in a simple regression is written as follows:

$$
y=\beta_{0}+\beta_{1} x+\varepsilon
$$

$\beta_{0}$ and $\beta_{1}$ correspond to the parameters of the population and $\varepsilon$ is a random variable called the error term. The error term takes into account the variability that is not explained by the linear relation between $x$ and $y$.

The patient population can be seen as the set of subpopulations related to a given value of $x$. Thus, one of the subpopulations consists of all patients that already reached the 60s. Each sub-population has a particular distribution of $y$. Thus a distribution of $y$ is associated with the patients that already reached the sixties. Each distribution of $y$ values has its own mean or mathematical expectation. The equation which describes how the average or the mathematical expectation of $y$, denoted $E(x)$, is related to $x$, is called the regression equation. The regression equation is written as follows:

$$
E(x)=\beta_{0}+\beta_{1} x
$$

$\beta_{0}$ and $\beta_{1}$ are unknown parameters. Subsequently, we will use the statistical procedure named the least squares estimation to estimate the values of $\beta_{0}$ and $\beta_{1}$. Sample statistics $b_{0}$ and $b_{1}$ are sample statistics used to estimate $\beta_{0}$ and $\beta_{1}$.

\section{The multiple regression}

Assuming the case where the Alzheimer's disease risk is predicted on the base of several predictors, for instance, the age of a patient, the geographical area, the number of work hour, the physical exercises 'hours, the existence of a parent with Alzheimer's disease, the feeding, and the existence of Lyme disease risk. The population undertaken in this study is a population of patients recorded in a dataset. The aim is to predict the percentage of Alzheimer's disease risk denoted on the base of the predictors pinpointed out above.

The Eq. (3) that describes the relation binding $x_{i}$ and $y$ with an error term denoted $\varepsilon$, corresponds to a regression model. The model used in a multiple regression is written as follows:

$$
y=\beta_{0}+\beta_{1} x_{1}+\beta_{2} x_{2}+\cdots+\beta_{k} x_{k}+\varepsilon
$$


The equation which describes how the average or the mathematical expectation of $y$, denoted $E(x)$, is related to $x_{i}$, is called the regression equation. The regression equation is written as follows:

$$
E(x)=\beta_{0}+\beta_{1} x_{1}+\beta_{2} x_{2}+\cdots+\beta_{k} x_{k}
$$

$\beta_{i \in[0, k]}$ are unknown parameters. Subsequently we will use the statistical procedure named the least squares estimation to estimate the values of $\beta_{i \in[0, k]}$. The statistics $b_{i \in[0, k]}$ are sample statistics used to estimate $\beta_{i \in[0, k]}$.

\section{The Alzheimer's disease prediction statistical model}

As we pointed out earlier in this paper, we believe that seven predictors have a great impact on predicting the Alzheimer's disease risk. The first predictor denoted $x_{1}$ is the age of an individual. The second predictor denoted $x_{2}$ is the geographical area. The third one denoted $x_{3}$ is the work's hours per a day. The fourth one denoted $x_{4}$ is the physical exercises' hours. The fifth one denoted $x_{5}$ is the existence of a parent with Alzheimer's disease. The sixth one denoted $x_{6}$ is the quality of feeding. The seventh and the last predictors denoted $x_{7}$ is the existence of Lyme disease. In conducting a statistical study, we would like to answer the following questions: do these variables really impact the Alzheimer's disease risk? Is there a relationship between the variables? If so, define this relationship. Can the values of these parameters be adjusted in order to efficiently predict the Alzheimer's disease risk?

Let assume that $x_{1 i}$ is the random variable associating the age to an individual $i . x_{2 i}$ is the random variable associating a number indicating an area to an individual $i . x_{3 i}$ is the random variable associating a number indicating the work's hours per a day to an individual $i . x_{4 i}$ is the random variable associating a number indicating the work's hours per a day to an individual $i . x_{5 i}$ is the random variable associating a number indicating the existence or absence of a parent with Alzheimer disease for an individual $i . x_{6 i}$ is the random variable associating a number indicating the quality of feeding of an individual $i . x_{7 i}$ is the random variable associating a number indicating the existence of Lyme disease for an individual $i$. The regression model that describes the studies is as follows:

$$
y=\beta_{0}+\beta_{1} x_{1}+\beta_{2} x_{2}+\beta_{3} x_{3}+\beta_{4} x_{4}+\beta_{5} x_{5}+\beta_{6} x_{6}+\beta_{7} x_{7}+\varepsilon
$$

Throughout this paper, the steps undertaken to estimate the unknown parameters $\beta_{i \in[0,7]}$ are explained in details. The next section explains the sampling stage.

\section{The Alzheimer's disease prediction sampling stage}

The sampling stage is a fundamental stage that has a great impact on the accuracy of the prediction model. Indeed, a small sample or a sample with similar individuals could lead to an inaccurate model [42]. Thus, sampling efficiently means predict efficiently. To tackle the problem of small samples a great number of statistical methods renowned for predicting efficiently based on small sample such as Hurvich and Tsai [47]. In addition, the central limit theorem could be applied when the population is large. This theorem states that the sampling distribution of the sample mean can be approximated by a normal probability distribution in the case of large sample. In practice, the sampling 
distribution can be approximated by a normal distribution when the sample size is greater than or equal to 30 [42].

To proceed the sampling stage, the proposed solution randomly picks up an individual. Then, compare it with the previously picked ones. If it is not similar or has close proprieties to any previously picked individual, it is added to the sample. The pseudo-code below details the steps token to accomplish the sampling stage.

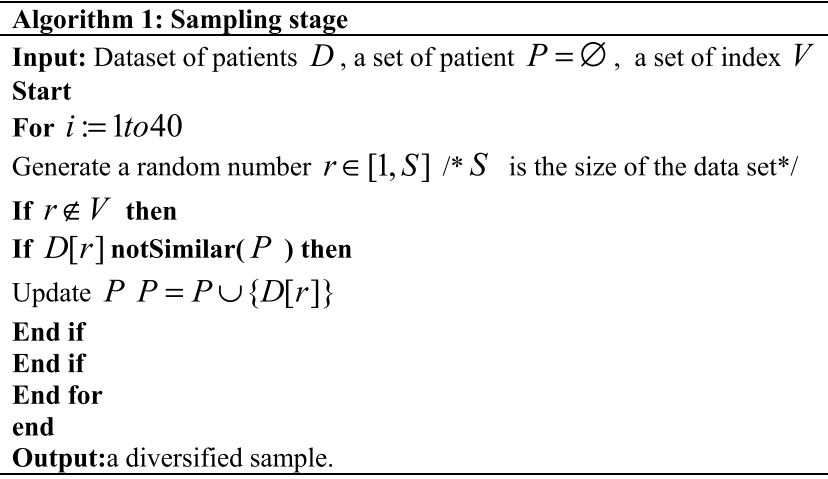

The function notsimilar takes a patient as an income and returns a Boolean as an outcome. The function compares each attribute of the income to the attributes of the sample if there is any similarity the function returns false. Otherwise it returns true.

\section{The Adjoint method for the least squares estimation problem}

To estimate the unknown parameters $\beta_{i \in[0, k]}$ Least Squares Estimation is the most common method used [20]. The $\mathrm{QR}$ factorization solve the problem of ordinary least squares [20]. The reference [20] relates step by step Least Squares Estimation method. Briefly, estimating the unknown parameters $\beta_{i \in[0, k]}$ is equivalent to solve $k$ equations system with $k$ unknowns. In our case and in contrast with the literature the Adjoint method is used to solve the k equations system. As a matter of fact, the system of equations can be expressed in a compact form by using matrix notation. The notation is as follows:

$$
A=\left[\begin{array}{cccc}
n & \sum_{i=1}^{n} x_{2 i} & \ldots & \sum_{i=1}^{n} x_{k i} \\
\sum_{i=1}^{n} x_{2 i} & \sum_{i=1}^{n} x_{2 i}^{2} & \ldots & \sum_{i=1}^{n} x_{2 i} x_{k i} \\
\vdots & \vdots & \ddots & \vdots \\
\sum_{i=1}^{n} x_{k i} & \sum_{i=1}^{n} x_{k i} x_{2 i} & \ldots & \sum_{i=1}^{n} x_{k i}^{2}
\end{array}\right], B=\left[\begin{array}{c}
b_{0} \\
b_{1} \\
\vdots \\
b_{k}
\end{array}\right], Y=\left[\begin{array}{c}
\sum_{i=1}^{n} y_{i} \\
\sum_{i=1}^{n} x_{2 i} y_{i} \\
\vdots \\
\sum_{i=1}^{n} x_{k i} y_{i}
\end{array}\right]
$$

where $n$ denotes the sample size and where: $A \cdot B=Y$ 


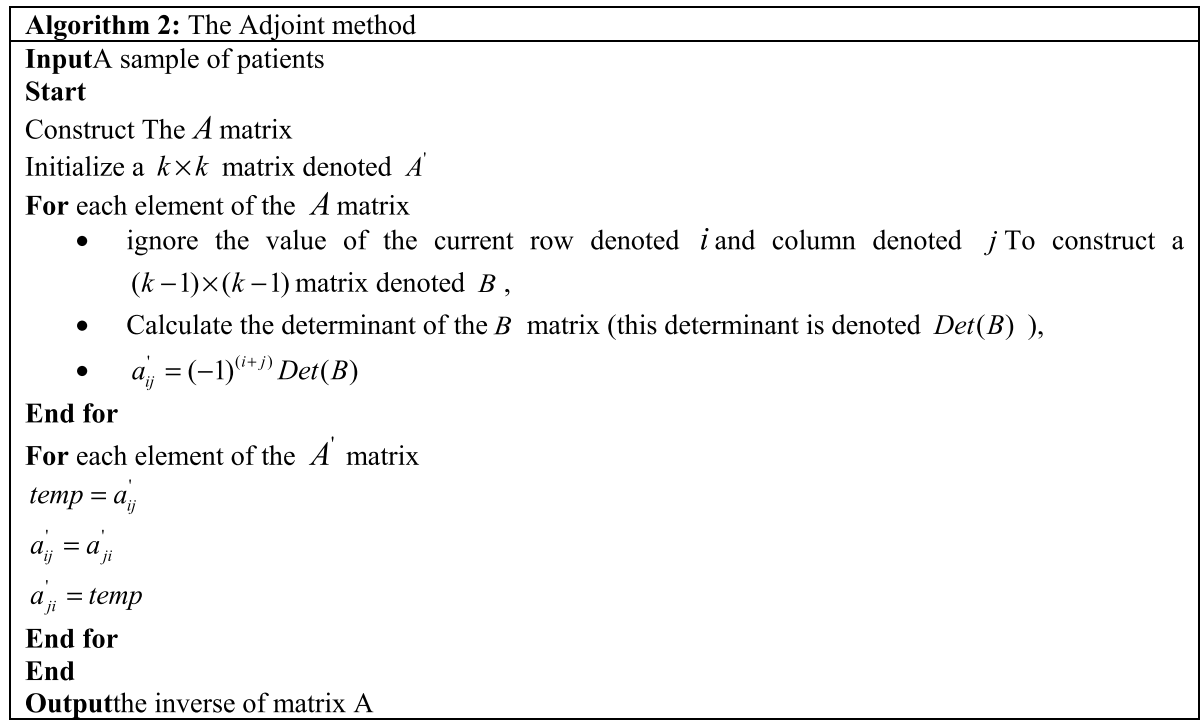

This part of code was tested against large scale data to discover its limits. Unfortunately, this method suffers from shortcomings when the patients 'sample is large and when the number of predictors is colossal. To overcome those shortcomings a new computational approach is presented thereafter. This method is massively parallel to absorb the massive calculations and to increase the method performance.

\section{MR-AM: MapReduce with Adjoint method}

MapReduce is a programming model for data processing [48]. It enables distributed algorithms in parallel on clusters of machines with varied features. MApReduce also handles the parallel computation issues thus the users deploy their efforts on programming model. Since its advent MapReduce has gained popularity in both scientific community and firms due to its effectiveness in parallel processing [49]. Indeed, the parallelization of QR factorization and SVD matrix decomposition methods is a relevant example of the scientific community interest toward MapReduce. The authors of Benson et al. [50] reported the matrix decomposition methods implemented on MapReduce programming. As pointed out earlier, the QR factorization is the most common method used to solve the least squares estimation problem. To the best of our knowledge, the Adjoint method has not been yet implemented on MapReduce framework. Thus, in this paper an implementation of Adjoint method on MapReduce is detailed in the aim to solve the least squares estimation problem.

Working within map reduce requires redesigning the traditional algorithms. As a matter of fact, the computation is expressed as two phases: Map and reduce. Each phase has key-value pairs as input and output. Two functions should also be specified: the map function and the reduce function. The types of key-value pairs may be chosen by the programmer.

A MapReduce-based Adjoint method (MR-AM) is proposed by this paper to make conventional Adjoint method work effectively in distributed environment. Our method has two steps. The following part describes in detail the two steps of our method. 
MapReduce breaks the processing into two phases: The map phase and the reduce phase. Each phase has (key, value) pairs as input and output. In the current study, a text input format represents each line in the dataset as a text value. The key is the first number departed by a plus sign from the reminder of the line. Consider the following sample lines of input data:

$$
\begin{array}{lcc}
0+067 & -011-95 \ldots \\
0+143 & -101-22 \ldots \\
1+243 & -011-22 \ldots \\
\vdots & \ddots & \vdots \\
\vdots & \ddots & \vdots \\
\vdots & \ddots & \vdots \\
4+340-310-12 \ldots \\
4+44-301-265 \ldots
\end{array}
$$

The keys is the line numbers of the $A$ matrix. The map function calculates the determinant for B matrix. The output of the Map function is as follows:

$$
\begin{aligned}
& (0,10) \\
& (0,22) \\
& (1,11) \\
& \vdots \ddots \vdots
\end{aligned}
$$

The pseudo code of Map Function is as follows:

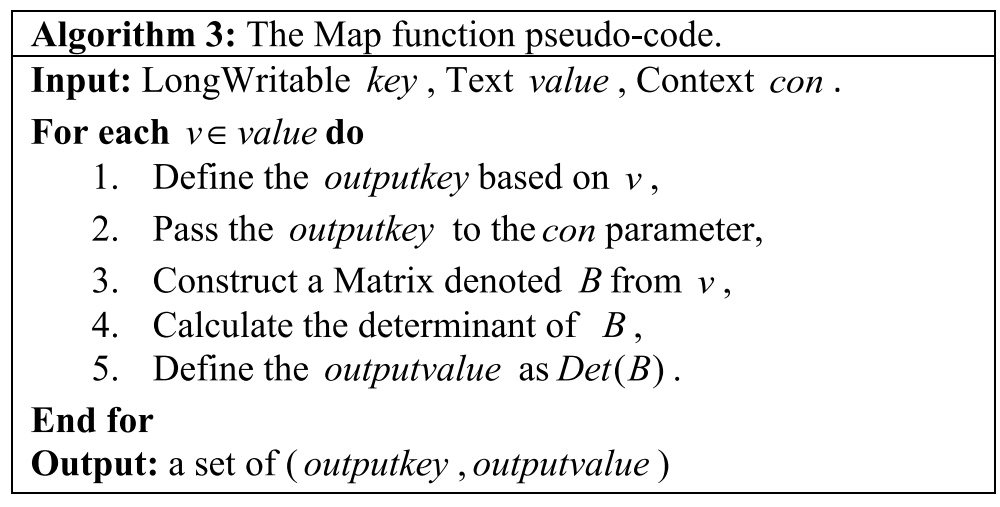

The output from the map function is processed by the MapReduce framework before being sent to the reduce function. This processing sorts and groups the key-value pairs by key. So, continuing the example, our reduce function sees the following input: 
$(0,[10,22])$

$(1,[11,111])$

$\vdots \ddots \vdots$

$\vdots \cdot \vdots$

$\vdots \because \vdots$

$(4,[78,80])$

The reduce function returns $\left(i, \beta_{i}\right)$ as output. The output of the reduce function is as follows:

$(0,2)$

$(1,5)$

$(2,6.5)$

$(3,7)$

$(4,8)$

The pseudo code of Reduce Function is as follows:

Algorithm 4: The Reduce function pseudo-code.

Input: Text word, Iterable $<$ IntWritable $>$ values, Context con

For each $v \in$ values do

1. $\operatorname{sum}=\operatorname{sum}+\frac{1}{\operatorname{Det}\left(X X^{\prime}\right)} \cdot(v \cdot Y X[i])$

2. $i++$;

End for

3. Define the outputkey as word variable.

4. The outputvalue as the sum variable.

Output: a set of $\left(j, \beta_{j}\right)$.

\section{Evaluation and experimental results}

In this section, we evaluate the accuracy and the performance of the proposed model on simulated data based on actual data of Riskalz dataset and of previous studies. To validate the resulting model and to evaluate its strength, the proposed solution involves additional steps that are detailed thereafter.

\section{Prediction accuracy measures}

The reduction of error (RE) assumes a central role in the verification procedure [51]. $\mathrm{RE}$ is an example of a forecast skill statistic. The forecast skill is defined as the relative 
accuracy of a set of forecasts which are usually the average values of the predictions. The equation used to calculate RE can be expressed in the following Eq. (6):

$$
R E=1-\frac{S S E_{v}}{S S E_{r e f}}
$$

where $S S E_{v}$ the sum of squares of validation errors between is observed and predicted values over the validation period and $S S E_{\text {ref }}$ is the sum of squares of validation errors between observed values and mean of the predictions often known as control values or reference values over the validation period. The difference between observed and predicted values is defined as validation error noted as $e(i)$. It can be mathematically expressed as Eq. (7).

$$
e(i)=Y_{i}-\hat{Y}(i)
$$

where $Y_{i}$ and $\hat{Y}(i)$ are the observed and predicted values of the predictions for validation data point $i$. The sum of the squares of errors for validation, $S S E_{v}$, can be expressed as Eq. (8) and the sum of squares of errors for reference, $S S E_{r e f}$, can be expressed as Eq. (9).

$$
\begin{aligned}
& S S E_{v}=\sum_{i=1}^{n_{v}} e_{(i)}^{2} \\
& S S E_{\text {ref }}=\sum_{i=1}^{n_{v}}\left(Y_{i}-\hat{Y}(i)\right)^{2}
\end{aligned}
$$

where $n_{v}$ denotes the total number of data points in the validation dataset and $\hat{Y}$ is the mean of the prediction, which usually serves as a reference or control value. Theoretically, the value of RE can range from negative infinity to one, where one indicates perfect prediction for the validation data set. It will only occur when all the residuals for validation data are zero. On the other hand, if $S S E_{v}$ is much greater than $S S E_{r e f}$ RE can be negative and large. A positive RE indicates that the regression model on average has some forecast skill. Contrastingly, if $\mathrm{RE} \leq 0$, the model is deemed to have no skill to predict. The similarity in form of the equations for RE and regression $R^{2}$ expressed as Eq. (10) suggests that RE can also be used as validation evidence for $R^{2}$. The closer the values of $\mathrm{RE}$ and $R^{2}$ are to each other, the more the model is accepted as a predictive tool.

$$
R^{2}=1-\frac{S S E}{S S E}=1-\frac{\sum_{i=1}^{n}\left(Y_{i}-\widehat{Y}_{i}\right)^{2}}{\sum_{i=1}^{n}\left(Y_{i}-\bar{Y}_{i}\right)^{2}}
$$

\section{Fisher's, Student's test and correlation coefficient}

Fisher's F-test, also called global significance test; is used to determine if there is a significant relationship between the dependent variable and the set of independent variables. However, Student's t test, called individual significance test, is used to determine whether each of the independent variables is significant. A Student test is performed for each model-independent variable. 


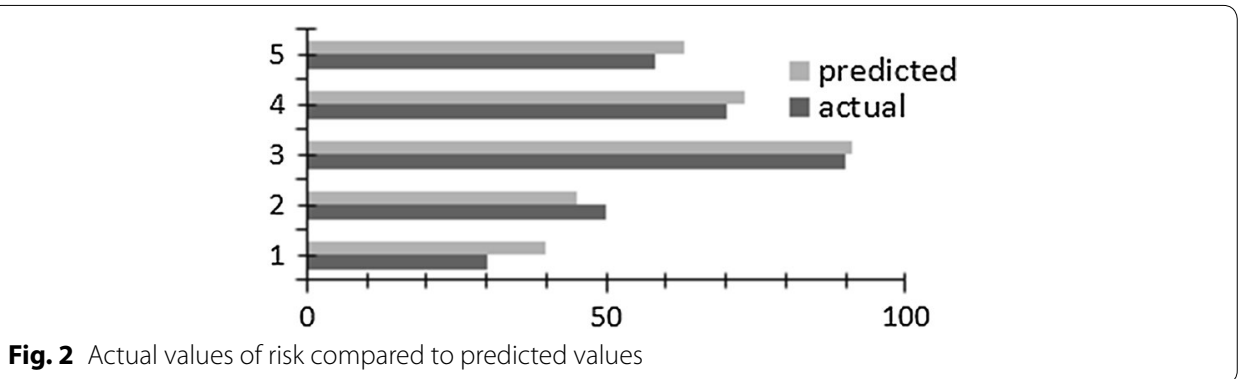

A correlation test is performed between the independent variables of the model. If the correlation coefficient between two variables is greater than 0.70 , it is not possible to determine the effect of a particular independent variable on the dependent variable.

A Fisher's test, based on Fisher's distribution, can be used to test whether a relationship is meaningful. With a single independent variable, the Fisher's test leads to the same conclusion as the Student test. On the other hand, with more than one independent variable, only the $\mathrm{F}$ test can be used to test the overall meaning of a relationship.

The logic underlying the use of the Fisher's test to determine whether the relationship is statistically significant or not, is based on the construction of two independent estimates of $\sigma^{2}$.

On the basis of the output model a punctual prediction is performed in this work. The Fig. 2 reports a comparison study carried out between the predicted value and the actual value of Alzheimer's disease risk.

\section{Experiments}

In this section, we test the proposed method on three datasets to confirm its robustness. For each case study, a brief description is given. At the end of this section, we carried out experiments and we compared the actual and predicted values for each case study.

\section{a. Student performance case study}

The dataset was collected by using school reports and questionnaires. The collected data approaches students achievement in secondary education of two Portuguese schools. The data attributes include student grades, demographic, social and school related features.

Two datasets are provided regarding the performance in two distinct subjects: Mathematics (mat) and Portuguese language (por).

In the current study, apply our approach to predict the G3 attributes on the basis of the reminder ones. An exhaustive list of attributes and their description could be found at http://archive.ics.uci.edu/ml/datasets/Student+Performance.

\section{b. Parkinsons telemonitoring case study}

The dataset is composed of a range of biomedical voice measurements from 42 people with early-stage Parkinson's disease recruited to a 6-month trial of a telemonitoring device for remote symptom progression monitoring. The recordings were automatically captured in the patient's homes. 
The main aim of the data is to predict the motor and total UPDRS scores ("motor UPDRS' and 'total_UPDRS') from the 16 voice measures. For more details readers could refers to https://archive.ics.uci.edu/ml/datasets/Parkinsons+Telemonitoring.

\section{c. The Levenson self report psychopathy scale value case study}

The data used to construct the prediction model is similar to the one used to spot sexual offenders available at http://reshare.ukdataservice.ac.uk/852521. Based on the factors provided in the studies of Ian Mitchell we aim to predict the value of the first and the second factors of LSRP measure. The following variable codes are relevant to aaFHNeyesAccuracyData, aaFHNeyesDwellTime and aaFHNeyesFix Count datasets:

- Participant = Identification number assigned to participant

- Eye tracker $=$ Method of eye tracking $(1=$ head mounted; $2=$ tower $)$

- Primary= Primary subscale of the Levenson Self Report Psychopathy Scale

- Secondary=Secondary subscale of the Levenson Self Report Psychopathy Scale

Variable names for each trial type are coded as follows [Emotion] [Intensity] [Sex] [Region] using the following values:

- Emotion: $\mathrm{ANG}=$ Angry expression, $\mathrm{DIS}=$ Disgust expression, $\mathrm{FEAR}=$ Fear expression, $\mathrm{HAP}=$ Happy expression, $\mathrm{SAD}=$ Sad expression, $\mathrm{SUR}=$ Surprise expression

- Intensity: $5=55,9=90$

- Sex: $\mathrm{F}=$ Female, $\mathrm{M}=$ male

- Region: Eyes = Eyes, Mouth = Mouth

Thus, ANG $5 \mathrm{~F}$ refers to an angry expression at $55 \%$ intensity, expressed by a female face and ANG $5 \mathrm{~F}$ Eyes refers to the eye region of the same face.

\section{d. The comparative study}

On the basis of the output model a punctual prediction is performed for each case study described above. The Figs. 3, 4, 5 report a comparison study carried out between the predicted value and the actual value of the predicted attribute.

\section{Discussion}

In this section we conducted a comparative study with the aim to position the proposed method within the methods solving the least square problem. Therefore, we use the Hadoop job performance model to estimate the job completion time given by Khan et al. [52].

In the current paper, we estimate the lower bound for a job with $N$ iterations. For this purpose, Hadoop benchmarks are used to estimate the inverse of read and write bandwidth respectively denoted $\beta_{r}$ and $\beta_{w}$. In addition, the limit number of maps and reduces, respectively denoted $m_{\max }$ and $r_{\text {max }}$, should be fixed in the Hadoop configuration. The Lower bound for a job with $N$ iterations, denoted $T_{l b}$, is estimated on the basis of the following formula: 
5
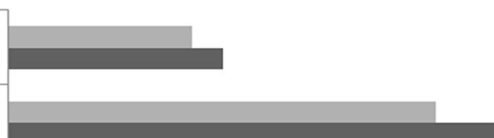

3

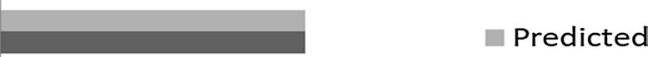

2

1

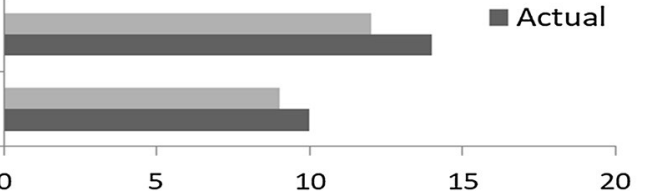

Fig. 3 Actual values of $\mathrm{G} 3$ compared to predicted values

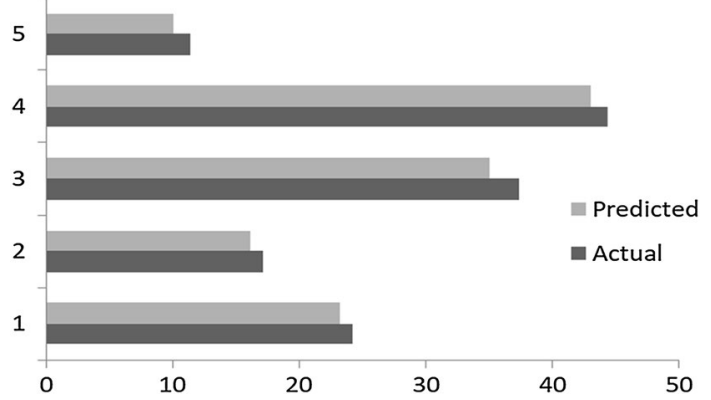

Fig. 4 Actual values of total_UPDRS compared to predicted values

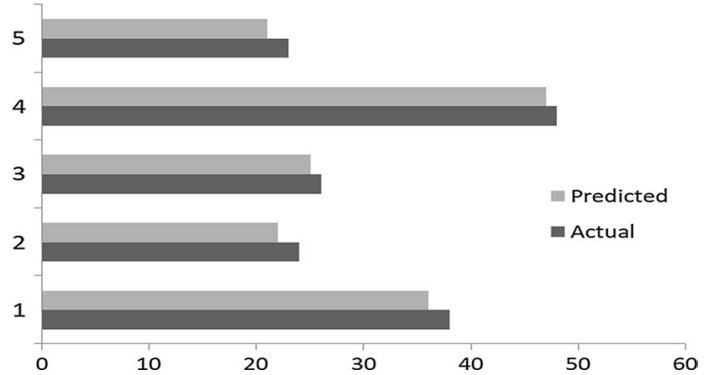

Fig. 5 Actual values of LSRP compared to predicted values

$$
T_{l b}=\sum_{j=1}^{N} \frac{R_{j}^{w} \beta_{r}+w_{j}^{m} \beta_{w}}{p_{j}^{m}}+\frac{R_{j}^{r} \beta_{r}+w_{j}^{r} \beta_{w}}{p_{j}^{r}}
$$

Subject to:

$$
\begin{gathered}
p_{j}^{m}=\min \left(m_{\max }, m_{j}\right) \\
p_{j}^{r}=\min \left(r_{\max }, r_{j}, k_{j}\right)
\end{gathered}
$$




$$
\begin{aligned}
& R_{j}^{m}=\text { number of data read in the jth map } \\
& R_{j}^{r}=\text { number of data read in the jth reduce } \\
& w_{j}^{m}=\text { number of data write in the jth map } \\
& w_{j}^{r}=\text { number of data write in the jth reduce }
\end{aligned}
$$

where $k_{j}$ is the number of distinct input keys passed to the reduce tasks for step $j$ and where $m_{j}$ and $r_{j}$ are respectively the number of map and reduce tasks for step $j$.

In the current subsection, we analyze the efficiency of the proposed MapReduce-based Adjoint method by comparing it with state-of-the-art of the parallelized factorization method such as:

1. Cholesky Benson et al. [50],

2. Indirect TSQR Benson et al. [50],

3. Direct TSQR Benson et al. [50],

4. Householder QR Benson et al. [50].

\begin{tabular}{|c|c|c|c|c|c|}
\hline & Cholesky (Bytes) & $\begin{array}{l}\text { Indirect TSQR } \\
\text { (bytes) }\end{array}$ & Direct TSQR (bytes) & $\begin{array}{l}\text { Householder } \\
\text { QR (bytes) }\end{array}$ & MR-AM (bytes) \\
\hline$R_{1}^{m}$ & $8 m n+K m$ & $8 m n+K m$ & $8 m n+K m$ & $8 m n+K m$ & $K m n+(m-1)(n-1)$ \\
\hline$W_{1}^{m}$ & $8 m_{1} n^{2}+8 m_{1} n$ & $8 m_{1} n^{2}+8 m_{1} n$ & $\begin{array}{l}8 m n+8 m_{1} n^{2} \\
+K m+64 m_{1}\end{array}$ & $8 m n+K m$ & $K m n+8 m n$ \\
\hline$R_{1}^{r}$ & $8 m_{1} n^{2}+8 m_{1} n$ & $8 m_{1} n^{2}+8 m_{1} n$ & 0 & 0 & $k m n+8 m n$ \\
\hline$W_{1}^{r}$ & $8 n^{2}+8 n$ & $8 r_{1} n^{2}+8 r_{1} n$ & 0 & 0 & $8 k+8 k$ \\
\hline$R_{2}^{m}$ & $8 n^{2}+8 n$ & $8 r_{1} n^{2}+8 r_{1} n$ & $8 m_{1} n^{2}+K m_{1}$ & $8 m n+K m$ & - \\
\hline$W_{2}^{m}$ & $8 n^{2}+8 n$ & $8 r_{1} n^{2}+8 r_{1} n$ & $8 m_{1} n^{2}+K m_{1}$ & $16 m_{1}$ & - \\
\hline$R_{2}^{r}$ & $8 n^{2}+8 n$ & $8 r_{1} n^{2}+8 r_{1} n$ & $8 m_{1} n^{2}+K m_{1}$ & 0 & - \\
\hline$W_{2}^{r}$ & $8 n^{2}+8 n$ & $8 n^{2}+8 n$ & $\begin{array}{c}8 m_{1} n^{2}+32 m_{1} \\
+8 n^{2}+8 n\end{array}$ & 0 & - \\
\hline$R_{3}^{m}$ & $\begin{array}{l}8 m n+K m \\
\quad+m_{3}\left(8 n^{2}+8 n\right)\end{array}$ & $\begin{array}{l}8 m n+K m \\
\quad+m_{3}\left(8 n^{2}+8 n\right)\end{array}$ & $\begin{array}{l}8 m n+K m \\
\quad+m_{3}\left(8 m_{1} n^{2}+64 m_{1}\right)\end{array}$ & - & - \\
\hline$W_{3}^{m}$ & $8 m n+K m$ & $8 m n+K m$ & $8 m n+K m$ & - & - \\
\hline$R_{3}^{r}$ & 0 & 0 & 0 & - & - \\
\hline$W_{3}^{r}$ & 0 & 0 & 0 & - & - \\
\hline
\end{tabular}

Table 1 The estimated $\beta_{r}$ and $\beta_{w}$ values for different HDFS file sizes

\begin{tabular}{lllll}
\hline HDFS Size (GB) & Write (MB/s) & Read (MB/s) & $\begin{array}{l}\boldsymbol{\beta}_{\boldsymbol{w}} \\
(\mathbf{s} / \mathbf{M B})\end{array}$ & $\begin{array}{l}\boldsymbol{\beta}_{\boldsymbol{r}} \\
\text { (s/MB) }\end{array}$ \\
\hline 1 & & & 0.015 & 0.017 \\
32 & 67.72 & 60.25 & 0.016 & 0.012 \\
64 & 61.39 & 85.91 & 0.012 & 0.012 \\
128 & 81.22 & 83.91 & 0.013 & 0.013 \\
\hline
\end{tabular}

Table 2 Number of reads and writes at each step (in bytes)

We assume a double is 8 bytes and is the number of bytes for a key. The amount of key data is separated from the amount of value data 
Table 3 The computed lower bounds $T_{l b}$ in seconds

\begin{tabular}{lllccc}
\hline HDFS size (GB) & Cholesky (s) & $\begin{array}{l}\text { Indirect } \\
\text { TSQR (s) }\end{array}$ & Direct TSQR (s) & $\begin{array}{l}\text { Householder } \\
\text { QR (s) }\end{array}$ & MR-AM (s) \\
\hline 32 & 802 & 802 & 1232 & 8224 & 625 \\
64 & 536 & 536 & 618 & 10,055 & 467 \\
128 & 366 & 366 & 10,475 & 30,994 & 450 \\
\hline
\end{tabular}

This set of algorithms represents is the set of parallel method based on MapReduce to solve the least square problem.

We conduct several groups of experiments on a local machine equipped with only 2 cores. To estimate $\beta_{r}$ and $\beta_{w}$, we used Hadoop benchmarks. Tables 1, 2 provides $\beta_{r}$ and $\beta_{w}$ values for different HDFS file sizes. Table 2 provides the number of reads and writes for state-of-the-art of the parallelized factorization methods and our proposed parallelized Adjoint algorithm. The computed lower bounds are contained in Table 3.

The Tables 2 and 3 confirms the performance of the proposed solution is competitive with existing methods in terms of number of operations and computational time.

\section{Conclusion}

In this paper, we carry out a comparative study between the parallel methods aiming to solve the least square estimation problem and our proposal. The results promote the use of the proposed method as the results confirm its efficiency and rapidity. Moreover, we presents a detailed description of the parallel MapReduce-based Adjoint method. The application of the method to predict the Alzheimer's disease risk confirms its robustness.

Authors' contributions

Authors propose a solution which receives a dataset of patients with a variable number of attributes and then constructs a statistical model to spot eventual Alzheimer's disease patients. Authors parallelize the Adjoint method via MapReduce to this aim. All authors read and approved the final manuscript.

Acknowledgements

Not applicable.

Competing interests

The authors declare that they have no competing interests.

Availability of data and materials

Not applicable.

Consent for publication

Not applicable.

Ethics approval and consent to participate

Not applicable.

Funding

Not applicable.

\section{Publisher's Note}

Springer Nature remains neutral with regard to jurisdictional claims in published maps and institutional affiliations.

Received: 4 May 2018 Accepted: 25 July 2018

Published online: 02 August 2018 


\section{References}

1. Li L, Ge RL, Zhou SM, Valerdi R. Guest editorial integrated healthcare information systems. IEEE Trans Inf Technol Biomed. 2012;16(4):515-7. https://doi.org/10.1109/TITB.2012.2198317.

2. Kumar A, Hancke GP. A Zigbee-based animal health monitoring system. IEEE Sens J. 2015;15(1):610-7. https://doi. org/10.1109/JSEN.2014.2349073.

3. Luke DA, Stamatakis KA. Systems science methods in public health: dynamics, networks, and agents. Annu Rev Public Health. 2012;33(1):357-76. https://doi.org/10.1146/annurev-publhealth-031210-101222.

4. Ferreira LK, Busatto GF. Neuroimaging in Alzheimer's disease: current role in clinical practice and potential future applications. Clinics. 2011;66(Suppl 1):19-24. https://doi.org/10.1590/S1807-59322011001300003.

5. Soucy JP, Bartha R, Bocti C, Borrie M, Burhan AM, Laforce R, Rosa-Neto P. Clinical applications of neuroimaging in patients with Alzheimer's disease: a review from the Fourth Canadian consensus conference on the diagnosis and treatment of Dementia 2012. Alzheimer's Res Ther. 2013;5(1):S3. https://doi.org/10.1186/alzrt199.

6. Thambisetty M, Lovestone S. Blood-based biomarkers of Alzheimer's disease: challenging but feasible. Biomarkers Med. 2010;4(1):65-79.

7. Lutz MW, Sundseth SS, Burns DK, Saunders AM, Hayden KM, Burke JR, Roses AD. A genetics-based biomarker risk algorithm for predicting risk of Alzheimer's disease. Alzheimer's Dementia Transl Res Clin Intervent. 2016;2(1):30-44. https://doi.org/10.1016/j.trci.2015.12.002

8. Liu-Ambrose T, Eng JJ, Boyd LA, Jacova C, Davis JC, Bryan S, Hsiung G-YR. Promotion of the mind through exercise (PROMOTE): a proof-of-concept randomized controlled trial of aerobic exercise training in older adults with vascular cognitive impairment. BMC Neurol. 2010;10(1):14. https://doi.org/10.1186/1471-2377-10-14.

9. Scarmeas $\mathrm{N}$, Luchsinger JA, Schupf $\mathrm{N}$, et al. Physical activity, diet, and risk of Alzheimer disease. JAMA. 2009;302(6):627-37. https://doi.org/10.1001/jama.2009.1144.

10. Nemati Karimooy H, Hosseini M, Nemati M, Esmaily HO. Lifelong physical activity affects mini mental state exam scores in individuals over 55 years of age. J Bodyw Mov Ther. 2012;16(2):230-5. https://doi.org/10.1016/j. jbmt.2011.08.003.

11. Winchester J, Dick MB, Gillen D, Reed B, Miller B, Tinklenberg J, Cotman CW. Walking stabilizes cognitive functioning in Alzheimer's disease (AD) across 1 year. Arch Gerontol Geriatr. 2013;56(1):96-103. https://doi.org/10.1016/j.archg er.2012.06.016.

12. Bu X-L, Yao X-Q, Jiao S-S, Zeng F, Liu Y-H, Xiang Y, Wang Y-J. A study on the association between infectious burden and Alzheimer's disease. Eur J Neurol. 2015;22(12):1519-25. https://doi.org/10.1111/ene.12477.

13. Maheshwari P, Eslick GD. Bacterial infection and Alzheimer's disease: a meta-analysis. J Alzheimer's Dis. 2015;43(3):957-66. https://doi.org/10.3233/JAD-140621.

14. MacDonald AB. Plaques of Alzheimer's disease originate from cysts of Borrelia burgdorferi, the Lyme disease spirochete. Med Hypotheses. 2006;67(3):592-600. https://doi.org/10.1016/j.mehy.2006.02.035.

15. Zettam M, Laassiri J, Enneya N. A software solution for preventing Alzheimer's disease based on MapReduce framework. In: 2017 IEEE international conference on information reuse and integration (IRI). San Diego, CA; 2017. p. 192-7. https://doi.org/10.1109/iri.2017.77.

16. Hastie T, Tibshirani R, Friedman J. The elements of statistical learning data mining, inference, and prediction. New York: Springer; 2009. https://doi.org/10.1007/978-0-387-84858-7_1.

17. Michie D, Spiegelhalter DJ, Taylor CC, Campbell J, editors. Machine learning, neural and statistical classification. Upper Saddle River: Ellis Horwood; 1994.

18. Tu JV. Advantages and disadvantages of using artificial neural networks versus logistic regression for predicting medical outcomes. J Clin Epidemiol. 1996;49(11):1225-31. https://doi.org/10.1016/S0895-4356(96)00002-9.

19. Hosmer DW, Lemeshow S. Applied logistic regression. 2nd ed. Hoboken: John Wiley \& Sons Inc;; 2005. https://doi. org/10.1002/0471722146.fmatter.

20. Rencher AC, Christensen WF. Methods of multivariate analysis. 3rd ed. Hoboken: Wiley; 2012.

21. Steyerberg EW. Clinical prediction models: a practical approach to development, validation, and updating. New York: Springer; 2008.

22. Lecleire S, Di Fiore F, Antonietti M, Ben Soussan E, Hellot M-F, Grigioni S, P Ducrotté. Undernutrition is predictive of early mortality after palliative self-expanding metal stent insertion in patients with inoperable or recurrent esophageal cancer. Gastrointest Endosc. 2006;64(4):479-84. https://doi.org/10.1016/j.gie.2006.03.930.

23. Janssen-Heijnen MLG, Houterman S, Lemmens V, Brenner H, Steyerberg EW, Coebergh JWW. Prognosis for longterm survivors of cancer. Ann Oncol. 2007;18(8):1408-13. https://doi.org/10.1093/annonc/mdm127.

24. Chatap NJ, Shrivastava AK. A survey on various classification techniques for medical image data. Int J Comput Appl. 2014;97(15):1-5.

25. Breiman L, Friedman J, Stone CJ, Olshen RA. Classification and regression trees. New ed. Boca Raton: Taylor \& Francis Ltd.; 1984.

26. Quinlan JR. Comparing connectionist and symbolic learning methods. In: Hanson SJ, Rivest RL, Drastal GA, editors Proceedings of a workshop on computational learning theory and natural learning systems: constraints and prospects, vol. 1. Cambridge: MIT Press; 1994. p. 445-56.

27. Kass GV. An exploratory technique for investigating large quantities of categorical data. J Roy Stat Soc Ser C (Appl Stat). 1980;29(2):119-27.

28. Lim T-S, Loh W-Y, Shih Y-S. A comparison of prediction accuracy, complexity, and training time of thirty-three old and new classification algorithms. Mach Learn. 2000;40(3):203-28. https://doi.org/10.1023/A:1007608224229.

29. Klecka WR. Discriminant analysis. 1st ed. Beverly Hills: SAGE Publications Inc.; 1980.

30. Hinton GE. How neural networks learn from experience. Sci Am. 1992;267(3):144-51

31. Rumelhart DE, Hinton GE, Williams RJ. Learning representations by back-propagating errors. Nature. 1986;323(6088):533-6. https://doi.org/10.1038/323533a0

32. Szolovits P, Patil RS, Schwartz WB. ARtificial intelligence in medical diagnosis. Ann Intern Med. 1988;108(1):80-7. https://doi.org/10.7326/0003-4819-108-1-80. 
33. Spelt L, Andersson B, Nilsson J, Andersson R. Prognostic models for outcome following liver resection for colorectal cancer metastases: a systematic review. Eur J Surg Oncol. 2012;38(1):16-24. https://doi.org/10.1016/j. ejso.2011.10.013.

34. Mortazavi D, Kouzani AZ, Soltanian-Zadeh H. Segmentation of multiple sclerosis lesions in MR images: a review. Neuroradiology. 2012;54(4):299-320. https://doi.org/10.1007/s00234-011-0886-7.

35. Ahmed FE. Artificial neural networks for diagnosis and survival prediction in colon cancer. Mol Cancer. 2005;4(1):29. https://doi.org/10.1186/1476-4598-4-29.

36. Bartosch-Härlid A, Andersson B, Aho U, Nilsson J, Andersson R. Artificial neural networks in pancreatic disease. Br J Surg. 2008;95(7):817-26. https://doi.org/10.1002/bjs.6239.

37. Siristatidis CS, Chrelias C, Pouliakis A, Katsimanis E, Kassanos D. Artificial neural networks in gynaecological diseases: current and potential future applications. Med Sci Monit Int Med J Exp Clin Res. 2010;16(10):RA231-6.

38. Shankaracharya DO, Samanta S, Vidyarthi AS. Computational intelligence in early diabetes diagnosis: a review. Rev Diabet Stud RDS. 2010;7(4):252-62. https://doi.org/10.1900/RDS.2010.7.252.

39. Amato F, López A, Peña-Méndez EM, Vaňhara P, Hampl A, Havel J. Artificial neural networks in medical diagnosis. J Appl Biomed. 2013;11(2):47-58. https://doi.org/10.2478/v10136-012-0031-x.

40. Cesa-Bianchi N, Lugosi G. Prediction, learning, and games. Cambridge: Cambridge University Press; 2006.

41. Chen Y, Crespi N, Ortiz AM, Shu L. Reality mining: a prediction algorithm for disease dynamics based on mobile big data. Inf Sci. 2017;379:82-93. https://doi.org/10.1016/j.ins.2016.07.075.

42. Anderson DR, Sweeney DJ, Williams TA, Camm JD, Cochran JJ. Statistiques pour l'économie et la gestion, 5e édition. De Boeck Universite; 2015

43. Tribout B. Statistiques pour économistes et gestionnaires. London: Pearson Education; 2008.

44. Tresch MC, Cheung VCK, d'Avella A. Matrix factorization algorithms for the identification of muscle synergies: evaluation on simulated and experimental data sets. J Neurophysiol. 2006;95(4):2199-212. https://doi.org/10.1152/ jn.00222.2005.

45. Giglio L, Kendall JD, Justice CO. Evaluation of global fire detection algorithms using simulated AVHRR infrared data. Int J Remote Sens. 1999;20(10):1947-85. https://doi.org/10.1080/014311699212290.

46. Murray RE, Ryan PB, Reisinger SJ. Design and validation of a data simulation model for longitudinal healthcare data. AMIA Ann Symp Proc. 2011;2011:1176-85.

47. Hurvich CM, Tsai C-L. Regression and time series model selection in small samples. Biometrika. 1989;76(2):297-307. https://doi.org/10.2307/2336663.

48. White T. Hadoop: the definitive guide. Farnham: O'Reilly Media Inc; 2009

49. Dean J, Ghemawat S. MapReduce: simplified data processing on large clusters. Commun ACM. 2008;51(1):107-13. https://doi.org/10.1145/1327452.1327492.

50. Benson AR, Gleich DF, Demmel J. Direct QR factorizations for tall-and-skinny matrices in MapReduce architectures. In: 2013 IEEE international conference on big data; 2013. p. 264-72. https://doi.org/10.1109/BigData.2013.6691583.

51. Lu P, Pei S, Tolliver D. Regression model evaluation for highway bridge component deterioration using national bridge inventory data. J Transp Res Forum. 2016;55(1):5-16.

52. Khan M, Jin Y, Li M, Xiang Y, Jiang C. Hadoop performance modeling for job estimation and resource provisioning. IEEE Trans Parallel Distrib Syst. 2016;27(2):441-54. https://doi.org/10.1109/TPDS.2015.2405552.

\section{Submit your manuscript to a SpringerOpen ${ }^{\circ}$ journal and benefit from:}

Convenient online submission

- Rigorous peer review

- Open access: articles freely available online

- High visibility within the field

- Retaining the copyright to your article

Submit your next manuscript at $\gg$ springeropen.com 